\title{
The role of imatinib mesylate (Glivec) for treatment of patients with malignant endocrine tumors positive for c-kit or PDGF-R
}

\author{
David J Gross ${ }^{1}$, Gabriel Munter ${ }^{1}$, Menachem Bitan ${ }^{2}$, Tali Siegal ${ }^{3}$, \\ Alberto Gabizon ${ }^{4}$, Ronny Weitzen ${ }^{5}$, Ofer Merimsky ${ }^{6}$, Aliza Ackerstein ${ }^{2}$, \\ Asher Salmon ${ }^{7}$, Avishai Sella ${ }^{8}$ and Shimon Slavin ${ }^{2}$ (The Israel Glivec in \\ Solid Tumors Study Group)
}

\footnotetext{
${ }^{1}$ Endocrinology and Metabolism Service, Department of Medicine, Hadassah University Hospital, P.O.B 12000, Jerusalem, Israel 91120

${ }^{2}$ Department of Bone Marrow Transplantation and Cancer Immunobiology Laboratory, Hadassah University Hospital, P.O.B 12000, Jerusalem, Israel 91120

${ }^{3}$ Gaffin Center for Neuro-Oncology, Hadassah University Hospital, P.O.B 12000, Jerusalem, Israel 91120

${ }^{4}$ Department of Oncology, Shaare Zedek Medical Center, Jerusalem, Israel

${ }^{5}$ Department of Oncology, Sheba Medical Center, Tel-Hashomer, Israel

${ }^{6}$ Department of Oncology, Sourasky Medical Center, Tel-Aviv, Israel

${ }^{7}$ Department of Oncology, Hadassah University Hospital, Jerusalem, Israel

${ }^{8}$ Department of Oncology, Assaf-Harofeh Medical Center, Zerifin, Israel
}

(Requests for offprints should be addressed to D J Gross; Email: gross@ vms.huji.ac.il)

\begin{abstract}
Imatinib mesylate (IM), a small molecule that is a selective inhibitor of the $A B L$, platelet derived growth factor receptor (PDGFR-R) and stem cell ligand receptor (c-kit) tyrosine kinases (TK). IM was also found to inhibit the TK activity of BCR/ABL fusion protein produced in chronic myelogenous leukemia, with marked clinical activity against the disease. Since both PDGF-R and c-kit both having a putative role in tumorigenesis, we investigated the efficacy and safety of the use of IM in patients with endocrine tumors unresponsive to conventional therapies that expressed c-kit and/or PDGF-R (within the framework of a comprehensive phase II multi-center study of IM in patients with solid tumors). IM was initiated at a dose of $400 \mathrm{mg} / \mathrm{day}$, with possible dose escalation within 1 week to $600 \mathrm{mg} /$ day and an option to raise the dose to $800 \mathrm{mg} /$ day in the event of progression and in the absence of safety concerns for a period of up to 12 months. Between September 2002 and July 2003, 15 adult patients with disseminated endocrine tumors were recruited as follows: medullary thyroid carcinoma (MTC, $n=6$ ); adrenocortical carcinoma (ACC, $n=4$ ); malignant pheochromocytoma (pheo, $n=2$ ); carcinoid (non-secreting, $n=2$ ), neuroendocrine tumor (NET, $n=1$ ). No objective responses were observed. MTC - disease progression in 4 patients, and treatment discontinuation in 2 patients due to adverse events; ACC - disease progression in 3 patients, and treatment discontinuation in 1 patient due to severe psychiatric adverse event; Pheo - disease progression in 2 patients; Carcinoid - stable disease in 1 patient (6.5 months), and disease progression in 1 patient; NET - disease progression in 1 patient. IM does not appear to be useful for treatment of malignant endocrine tumors, also causing significant toxicity in this patient population.
\end{abstract}

Endocrine-Related Cancer (2006) 13 535-540

\section{Introduction}

Imatinib mesylate (IM), is a small molecule selective inhibitor of the c-ABL, platelet derived growth factor receptor (PDGFR-R) and stem cell ligand receptor (c-kit) tyrosine kinases (TK) (Mol et al. 2004). IM was also found to inhibit the TK activity of $\mathrm{BCR} / \mathrm{Abl}$ fusion protein produced in 
chronic myelogenous leukemia (CML). Subsequently, in clinical trials IM was found to be highly effective in patients with CML, featuring unprecedented hematological, cytogenetic and molecular responses; IM has become the treatment of choice for such patients (Druker 2003). Both c-kit and PGDFR have been shown to play important roles in oncogenesis in a broad spectrum of hematological and solid tumors (Fletcher 2004). Moreover, occasional patients with solid tumors expressing c-kit and/or PDGFR have also shown responses to IM. This is particularly striking in patients with c-kit positive gastrointestinal stromal tumors (GIST) for which IM has become standard therapy in inoperable cases (Connolly et al. 2003, Eisenberg \& von Mehren 2003). IM has also proved to be useful in the cutaneous malignant tumor dermatofibrosarcoma protuberans, in those cases associated with a translocation between chromosomes 17 and 22 that places the plateletderived growth factor-B under the control of the collagen 1A1 promoter (McArthur et al. 2005). These observations prompted us to design a multicenter national study for patients with solid tumors, other than GIST, expressing either c-kit and/or PDGFR to evaluate the clinical response to IM. In this paper, we report the results of IM in patients with cancers of the endocrine system, within the framework of the more comprehensive national study.

\section{Patients and methods}

\section{Patients and eligibility criteria}

This was a non-randomized, open label, Phase II, dose escalating study performed at the HadassahHebrew University Medical Center (Jerusalem, Israel) within the framework of a investigator initiated, comprehensive multi-center study assessing the effect of glivec in patients with a variety of solid tumors. The Local Research Ethics Committee at each of the participating institutions and the Israeli Ministry of Health approved the study, all patients gave written, informed consent. All of the patients entered into this study had unresectable and/or metastatic endocrine cancers, pathologically verified, and therefore incurable with any conventional multimodality approach. All patients had immunohistochemical documentation of c-kit and/or PDGFR expression in the primary tumor or metastases by a central laboratory (Oncotest, Netanya, Israel). Eligible patients were those with a life expectancy of at least 3 months; age $>18$ years; ECOG performance status 0-3; no chemotherapy, immunotherapy, or radiotherapy within 4 weeks of entering the study; at least one measurable site of disease; adequate organ function (absolute neutrophil count $>1.5 \times 10^{9} / 1$, platelets $\left.>100 \times 10^{9} / 1\right)$, total serum bilirubin $<\times 1.5$ the upper limit of normal, serum alanine aminotransferase and aspartate aminotransferase $<\times 2.5$ upper limit of normal or $<\times 5$ the upper limit of normal if hepatic metastases were present and serum creatinine $<\times 1.5$ of the upper limit of normal. Patients with cerebral metastases or pregnant women were excluded.

\section{Treatment administration}

Pretreatment evaluation included a complete history and clinical examination, full blood count, biochemical profile, coagulation profile and pregnancy test for women of childbearing age. CT or MRI studies to evaluate sites of disease were performed up to 4 weeks before starting chemotherapy. Serum calcitonin and CEA levels were also ascertained prior to therapy in patients with medullary thyroid carcinoma (MTC) and urinary catecholamines in patients with malignant pheochromocytoma (Pheo). IM was supplied to the study investigators by Novartis as $100 \mathrm{mg}$ capsules packaged in polyethylene bottles. Patients received IM orally $400 \mathrm{mg} /$ day for an exposure period of up to 12 months, providing that the patient was considered to benefit from the treatment and the absence of safety concerns. In the event of lack of response, the dosage could be increased to $600 \mathrm{mg} /$ day with the option to increase dosage to $400 \mathrm{mg}$ twice daily. Patients were instructed to take the medication with breakfast in the sitting position together with a large glass of water to prevent local irritation and to refrain from caffeine or grapefruit containing foods that affect drug absorption.

\section{Evaluation of toxicity and dose escalation}

Drug toxicity was graded using National Cancer Institute Common Toxicity Criteria version 2.0. Toxicity assessment, full physical examination, full blood count, and a biochemical profile were performed weekly during the first month of the study, bi-weekly during the second month and subsequently on a monthly basis for the duration of the study (one year). The algorithms for dose modifications in the event of hematological or 
Table 1 Patient characteristics

\begin{tabular}{|c|c|c|c|c|c|c|c|}
\hline Patient no. & Gender & Age & Tumor type & Tumor sites & Disease status & PDGF-R & $c-k i t$ \\
\hline 1 & M & 18 & MTC (MEN2b) & Neck, lungs, liver & SD & + & + \\
\hline 2 & $\mathrm{~F}$ & 62 & MTC & Liver & SD & + & + \\
\hline 3 & M & 71 & MTC & Neck, lungs, mediastinum & PD & + & + \\
\hline 4 & $\mathrm{~F}$ & 32 & MTC & Neck, bone & PD & + & - \\
\hline 5 & M & 52 & MTC & Neck, lung, bone & PD & + & + \\
\hline 6 & $\mathrm{~F}$ & 28 & MTC & Neck, lungs & PD & - & + \\
\hline 7 & $\mathrm{~F}$ & 44 & ACC & Lungs, liver, adrenal bed & PD & n.d. & + \\
\hline 8 & $\mathrm{~F}$ & 34 & $\mathrm{ACC}$ & Lungs, liver, adrenal bed & PD & + & + \\
\hline 9 & $\mathrm{~F}$ & 49 & ACC & Lungs, liver, adrenal bed & PD & + & - \\
\hline 10 & M & 63 & ACC & Lungs, liver, mediastinum & PD & + & + \\
\hline 11 & $\mathrm{~F}$ & 56 & carcinoid & Liver, retroperitoneum & PD & + & + \\
\hline 12 & M & 55 & carcinoid & Lung, pericard, pleura & SD & & \\
\hline 13 & $\mathrm{~F}$ & 18 & islet cell & Pancreas, liver & PD & - & + \\
\hline 14 & M & 58 & pheochromocytoma & Lungs, liver, retroperitoneum & PD & + & - \\
\hline 15 & M & 25 & pheochromocytoma & Retroperitoneum & PD & + & + \\
\hline
\end{tabular}

PDGFR-R, platelet derived growth factor receptor; SD, stable disease; PD, progressive disease; n.d., not detected.

non-hematological toxicity are available from the authors upon request.

\section{Disease evaluation and response assessment}

Tumor assessments consisted of radiological evaluations (computed tomography scan of disease sites); determination of serum calcitonin and CEA levels in MTC patients; urinary catecholamine determinations in pheochromocytoma patients and clinical assessments. These tests were performed before starting treatment and after three months of therapy. Patients who received treatment for at least three months were evaluable for response. In addition, patients who developed rapid tumor progression or died of progressive disease before the three-month period were considered evaluable for response. Responses to treatment were defined using the WHO criteria.

\section{Results}

Fifteen patients with endocrine cancers were recruited into the study. Patient characteristics at baseline are summarized in Table 1. Tumor types (number of patients in parenthesis): MTC (6), ACC (4), carcinoid tumors (2), Pheo (2) and pancreatic neuroendocrine tumor (1). All but three patients had radiological evidence of progressive disease. Two patients had received at least one previous chemotherapy regimen for advanced disease, two patients received experimental treatment with thalidomide and one MTC patient received experimental radio-immunotherapy with ${ }^{131}$ I-anti-CEA antibodies. All patients were started on the initial dose of $400 \mathrm{mg}$ IM, four patients reached a dosage of $600 \mathrm{mg} /$ day and six patients $800 \mathrm{mg} /$ day. Treatment duration for each disease is detailed in Table 2. In five patients the drug was

Table 2 Results of treatment

\begin{tabular}{|c|c|c|c|c|c|c|c|}
\hline & \multirow[b]{2}{*}{$n$} & \multicolumn{2}{|c|}{ Treatment duration (months) } & \multirow{2}{*}{$\begin{array}{l}\text { Complete } \\
\text { remission }\end{array}$} & \multirow{2}{*}{$\begin{array}{l}\text { Partial } \\
\text { remission }\end{array}$} & \multirow{2}{*}{$\begin{array}{l}\text { Stable } \\
\text { disease }\end{array}$} & \multirow{2}{*}{$\begin{array}{c}\text { Progressive } \\
\text { disease }\end{array}$} \\
\hline & & mean \pm S.D. & range & & & & \\
\hline \multicolumn{8}{|l|}{ Tumor type } \\
\hline MTC & 6 & $3.4 \pm 2.5$ & $1-6$ & 0 & 0 & 2 & 4 \\
\hline ACC & 4 & $4.9 \pm 3$ & $1-7.5$ & 0 & 0 & 0 & 4 \\
\hline carcinoid & 2 & 6 & 6 & 0 & 0 & 1 & 1 \\
\hline pheochromocytoma & 2 & $4 \pm 0.7$ & $4-5$ & 0 & 0 & 0 & 2 \\
\hline NET & 1 & 3 & 3 & 0 & 0 & 0 & 1 \\
\hline
\end{tabular}


Table 3 Toxicity (adverse effects) of imatinib mesylate

\begin{tabular}{lc}
\hline & No. (\%) \\
\hline NCI CTC(v 2.0) grading & \\
Grade 3-4 & $3(20)$ \\
Bleeding tendency & $1(7)$ \\
Suicide attempt & \\
Grade 1-2 & $11(73)$ \\
Fatigue & $8(53)$ \\
Anorexia & $6(40)$ \\
Nausea & $4(26)$ \\
Bleeding tendency & \\
\hline
\end{tabular}

discontinued prior to the initial 3 months evaluation point due to major side effects (Table 3 ). None of the patients showed an objective anatomical tumor response. One MTC patient displayed an apparent acceleration of tumor growth under IM and developed superior vena cava syndrome. In three patients who had slow growing disease at study entry, the disease remained stable. In the remainder of the patients disease progression was unrelenting despite IM treatment. In the two pheochromocytoma patients, urinary catecholamines levels
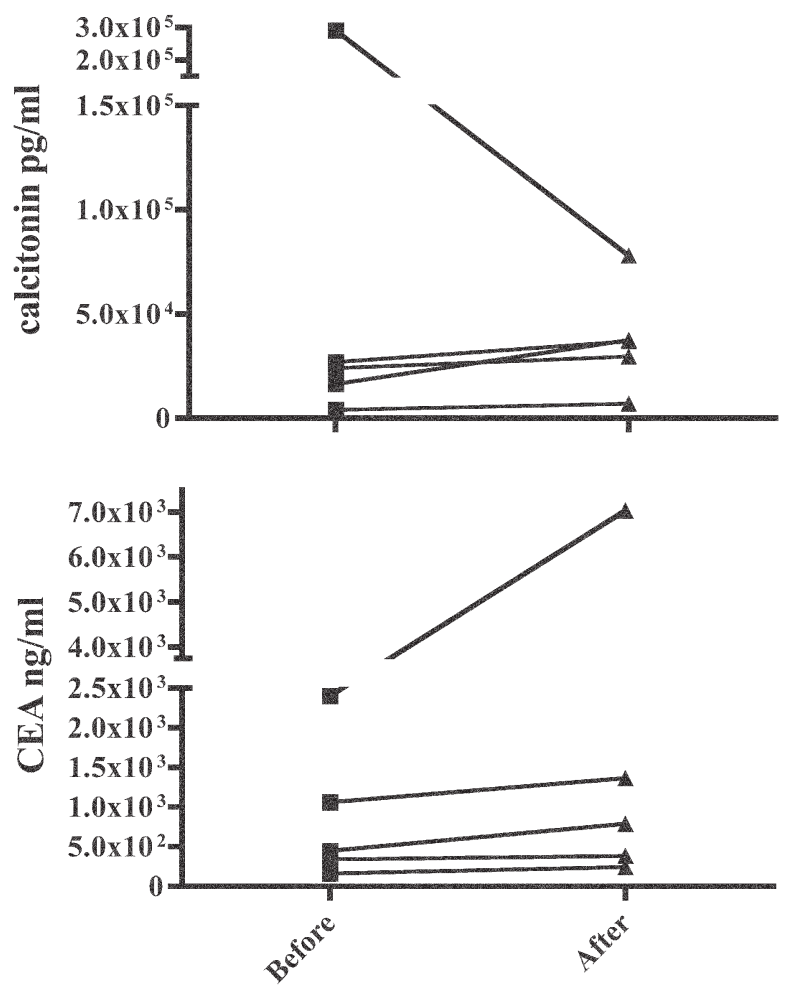

Figure 1 The effect of IM on pre and post-treament serum levels of calcitonin and carcinoembryonic antigen (CEA) in five patients with MTC were determined prior to and after therapy and were not affected by IM treatment (data not shown). In the patients with MTC pre and post treatment levels of serum calcitonin and carcinoembryonic antigen (CEA) were determined (Fig. 1): in 1 out of 5 patients, a marked decrease in calcitonin level was noted, however in the same patient the CEA level increased. In the remainder of the patients either no change or a slight increase in calcitonin and CEA levels was noted. We noted an unexpected frequency of side effects in our patients (Table 3): nausea, anorexia, fatigue and diarrhea were common and did not lead to withdrawal from the study; tendency to bleed was noted in: five patients with MTC (one of which developed disseminated intravascular coagulation that subsided with drug withdrawal); one patient with ACC and one pheochromocytoma patient. One patient with ACC attempted suicide leading to drug discontinuation. All 4 patients who had grade 3-4 adverse effects (Table 3) were withdrawn from the study.

\section{Discussion}

In this study, we examined the effect of IM on progression of disease in a group of patients with metastatic endocrine cancers, within the framework of a comprehensive multi-center study on patients with c-kit/PDGF-R positive solid tumors. Due to the small number of patients in the group as a whole and within each category, and the relatively short duration of treatment due to adverse effects, it is clear that statistically significant conclusions cannot be drawn from this study. However, in view of the rarity of the tumors examined, and the lack of any prior information regarding the effect of IM in these diseases, we believe it is important to report the results of this study.

The largest sub-group of patients treated with IM had MTC. This thyroid neuroendocrine cancer is frequently associated with mutations causing constitutive activation of the ret proto-oncogene tyrosine kinase both in premalignant lesions (C-cell hyperplasia) and frank MTC. IM has recently been shown to inhibit ret proto-oncogene tyrosine kinase activity in an MTC cell line in vitro (Cohen et al. 2002, Skinner et al. 2003), together with a decrease of either cell replication (Cohen et al. 2002) or viability (Skinner et al. 2003). Moreover, in the related neuroendocrine tumor neuroblastoma (NBL), IM has recently been shown to inhibit the growth of tumor cells in vitro and in vivo, 
associated with suppression of PDGF-R and c-kit phosphorylation (Beppu et al. 2004). Thus, preclinical evidence suggested a possible beneficial role for IM in MTC patients. In this clinical trial, we did not observe any beneficial effect in any of the MTC patients, in fact there was a significant occurrence of side effects. One patient had rapid tumor growth leading to superior vena cava syndrome, suggesting acceleration of his disease under therapy. The putative anti-cancer effect of inhibition of PDGF-R activity is thought to be due to an antiangiogenic effect, thus depriving the tumor of its oxygen supply (Adams et al. 2002). However, it has recently been shown that tumor oxygen deprivation can be a double-edged sword, since hypoxia induced signaling via the $c$-Met in tumor cells can result in a more aggressive tumor phenotype (Bottaro \& Liotta 2003). Since $c$-Met is expressed in some MTC tumors (Papotti et al. 2000), it is quite possible that the potential anti-tumor effect of IM in MTC is offset by hypoxia-enhanced aggressiveness of the tumor phenotype. A possible solution to this problem is to treat with a $c$-Met inhibitor in addition to IM.

Pheochromocytoma and NBL are closely related tumors of adrenomedullary origin, both secreting catecholamines. In fact, some malignant pheochromocytomas have a histological appearance similar to NBL, as was apparent in one of the two pheochromocytoma patients enrolled in this study. Expression of $c$-kit has been shown in both normal and neoplastic adrenomedullary tissue (Matsuda et al. 1993). $c$-kit has been shown to be a survival factor in NBL (Timeus et al. 1997, 2001) and could play a similar role in pheochromocytoma. IM therefore would appear to be of potential therapeutic value in these patients. We did not see a beneficial effect of IM on tumor progression in the two pheochromocytoma patients. However, a ${ }^{123}$ I-metaiodobenzylguanidine $\left({ }^{123} \mathrm{I}-\mathrm{MIBG}\right)$ scan that was negative in one patient prior to IM therapy became positive subsequent to treatment, allowing effective treatment of this patient with high-dose ${ }^{131} \mathrm{I}-\mathrm{MIBG}$; in the other patient a pretreatment somatostatin receptor scintigraphy (Octreoscan) became positive subsequent to therapy. These observations are of potential clinical utility and warrant further investigation.

$\mathrm{ACC}$ is a rare endocrine tumor originating from the adrenal cortex. This tumor has a dismal prognosis with less than $30 \%$ of patients surviving for five years (Wajchenberg et al. 2000). The potential roles of $c$-kit and/or PDGF-R in ACC evolution are unknown. However, some patients show $c$-kit expression in the tumor (Gross DJ, unpublished observations). In view of the dismal prognosis, patients with either $c$-kit or PDGF-R expression were considered candidates for IM. We did not, however, observe any beneficial effect of IM in the four ACC patients treated with IM.

Carcinoid tumors originate from the enterochromaffin cells in the gut or bronchi. Most tumors $(\sim 75 \%)$ are of midgut origin and are located in the distal ileum. Approximately $40-70 \%$ of cases present with multicentric disease. In these cases the five-year survival is approximately 38\% (Modlin et al. 2003). Carcinoids have been shown to express the PDGF system to a high degree. PDGF-R was found to be expressed on tumor cells and stroma in $70 \%$ of tissues examined (Funa et al. 1990). These findings suggest that PDGF may be involved in the autocrine stimulation of tumor cells and stimulation of stromal cell growth through paracrine and possibly autocrine mechanisms. Thus, IM-induced inhibition of PDGFR function could be of benefit in these patients. In a preliminary report, IM was found to be of benefit in patients with carcinoid tumor (Oberg 2003), however in a study of 31 patients, 21 of which were concurrently treated with octreotide, only a marginal benefit was observed (Carr et al. 2004). In the two carcinoid patients and an additional patient with a pancreatic neuroendocrine tumor in this study, we did not observe any beneficial effect of IM.

In summary, in this pilot study of IM in patients with endocrine malignancies, we did not discern a beneficial effect on the course of the disease. Our findings are in keeping with recent reports on the effect of IM in patients with small cell lung carcinoma, a cancer with a related neuroendocrine phenotype (Gambacorti-Passerini et al. 2004, Dy et al. 2005, Krug et al. 2005). The lack of efficacy in our study might have been due, in part, to the high frequency of adverse effects that required early cessation of therapy in many of the patients, which could reflect our inclusion of patients with advanced disease, Eastern Cooperative Oncology Group Performance Status (ECOG PS) 0-3, while most phase II studies are restricted to PS 0-2. Further studies on the effect of IM in our patient group may be warranted in conjunction with other therapeutic modalities, such as $c$-MET inhibition.

\section{Acknowledgements}

We are grateful to Novartis for the donation of imatinib mesylate (Glivec). 


\section{Funding}

The authors declare that there is no conflict of interest that would prejudice the impartiality of this scientific work.

\section{References}

Adams J, Huang P \& Patrick D 2002 A strategy for the design of multiplex inhibitors for kinase-mediated signalling in angiogenesis. Current Opinion in Chemical Biology 6 486-492.

Beppu K, Jaboine J, Merchant MS, Mackall CL \& Thiele CJ 2004 Effect of imatinib mesylate on neuroblastoma tumorigenesis and vascular endothelial growth factor expression. Journal of the National Cancer Institute 96 46-55.

Bottaro DP \& Liotta LA 2003 Cancer: Out of air is not out of action. Nature 423 593-595.

Carr K, Yao JC, Rashid A, Yeung S-C, Szklaruk J, Baker J, Vauthy J-N, Curley S, Ellis L \& Ajani JA 2004 A phase II trial of imatinib in patients with advanced carcinoid tumor. Journal of Clinical Oncology 224124.

Cohen MS, Hussain HB \& Moley JF 2002 Inhibition of medullary thyroid carcinoma cell proliferation and RET phosphorylation by tyrosine kinase inhibitors. Surgery 132 960-966; discussion 966-967.

Connolly BL \& von Mehren M 2003 Pharmacotherapy of gastrointestinal stromal tumours. Expert Opinions in Pharmacotherapy 4 869-874.

Connolly EM, Gaffney E \& Reynolds JV 2003 Gastrointestinal stromal tumours. British Journal of Surgery 90 1178-1186.

Druker BJ 2003 Imatinib mesylate in the treatment of chronic myeloid leukaemia. Expert Opinion in Pharmacotherapy 4 963-971.

Dy GK, Miller AA, Mandrekar SJ, Aubry MC, Langdon RM, Jr., Morton RF, Schild SE, Jett JR \& Adjei AA 2005 A phase II trial of imatinib (ST1571) in patients with c-kit expressing relapsed small-cell lung cancer: a CALGB and NCCTG study. Annals in Oncology 16 1811-1816.

Fletcher JA 2004 Role of KIT and platelet-derived growth factor receptors as oncoproteins. Seminars in Oncology 31 4-11.

Funa K, Papanicolaou V, Juhlin C, Rastad J, Akerstrom G, Heldin CH \& Oberg K 1990 Expression of platelet-derived growth factor beta-receptors on stromal tissue cells in human carcinoid tumors. Cancer Research 50 748-753.

Gambacorti-Passerini C, Piazza R, Tornaghi L, Pilotti S \& Pogliani E 2004 Development of c-Kit-expressing smallcell lung cancer in a chronic myeloid leukemia patient during imatinib treatment. Journal of the National Cancer Institute 96 1723-1724.

Krug LM, Crapanzano JP, Azzoli CG, Miller VA, Rizvi N, Gomez J, Kris MG, Pizzo B, Tyson L, Dunne M et al. 2005 Imatinib mesylate lacks activity in small cell lung carcinoma expressing c-kit protein: a phase II clinical trial. Cancer 103 2128-2131.

McArthur GA, Demetri GD, van Oosterom A, Heinrich MC, Debiec-Rychter M, Corless CL, Nikolova Z, Dimitrijevic S \& Fletcher JA 2005 Molecular and clinical analysis of locally advanced dermatofibrosarcoma protuberans treated with imatinib: Imatinib Target Exploration Consortium Study B2225. Journal of Clinical Oncology 23 866-873.

Matsuda R, Takahashi T, Nakamura S, Sekido Y, Nishida K, Seto M, Seito T, Sugiura T \& Ariyoshi Y 1993 Expression of the c-kit protein in human solid tumors and in corresponding fetal and adult normal tissues. American Journal of Pathology 142 339-346.

Modlin IM, Lye KD \& Kidd M 2003 A 5-decade analysis of 13,715 carcinoid tumors. Cancer 97 934-959.

Mol CD, Dougan DR, Schneider TR, Skene RJ, Kraus ML, Scheibe DN, Snell GP, Zou H, Sang BC \& Wilson KP 2004 Structural basis for the autoinhibition and STI-571 inhibition of c-Kit tyrosine kinase. Journal of Biological Chemistry 279 31655-31663.

Oberg K 2003 Diagnosis and treatment of carcinoid tumors. Expert Reviews in Anticancer Therapy 3 863-877.

Papotti M, Olivero M, Volante M, Negro F, Prat M, Comoglio PM \& DiRenzo MF 2000 Expression of hepatocyte growth factor (HGF) and its receptor (MET) in medullary carcinoma of the thyroid. Endocrine Pathology 11 19-30.

Skinner MA, Safford SD \& Freemerman AJ 2003 RET tyrosine kinase and medullary thyroid cells are unaffected by clinical doses of STI571. Anticancer Research 23 3601-3606.

Timeus F, Crescenzio N, Valle P, Pistamiglio P, Piglione M, Garelli E, Ricotti E, Rocchi P, Strippoli P, Cordero di Montezemolo L et al. 1997 Stem cell factor suppresses apoptosis in neuroblastoma cell lines. Experimental Hematology 25 1253-1260.

Timeus F, Ricotti E, Crescenzio N, Garelli E, Doria A, Spinelli M, Ramenghi U \& Basso G 2001 Flt-3 and its ligand are expressed in neural crest-derived tumors and promote survival and proliferation of their cell lines. Laboratory Investigations 81 1025-1037.

Wajchenberg BL, Albergaria Pereira MA, Medonca BB, Latronico AC, Campos Carneiro P, Alves VA, Zerbini MC, Liberman B, Carlos Gomes G \& Kirschner MA 2000 Adrenocortical carcinoma: clinical and laboratory observations. Cancer 88 711-736. 